\title{
Características e produção científica dos grupos de pesquisa do CNPq/DGP nas áreas de Ciência da Informação e Museologia (1992 - 2012)
}

Jonathan Rosa Moreira

Graduado em Bacharelado em Sistemas de Informação pela Faculdade Cenecista de Brasília Campanha Nacional de Escolas da Comunidade MBA em Governança em Tecnologia da Informação pelo Centro Universitário do Maranhão. Mestre em Gestão do Conhecimento e da Tecnologia da Informação na Universidade Católica de Brasília Doutorando em Ciência da Informação na Universidade de Brasília

Jayme Leiro Vilan Filho,

Professor da UnB. Doutor em Ciência da Informação. Mestre em Biblioteconomia e Documentação com graduação em Processamento de Dados Membro do Grupo de Pesquisa de Comunicação Científica (PPGCInf/UnB)

Suzana Pinheiro Machado Mueller

Graduada em Biblioteconomia e Documentação pela Universidade Federal do Paraná Master in Library Science pela George Peabody College For Teachers (Nashville, Tenn, EUA).Doutora (PhD) em Information Studies pela University of Sheffield (Inglaterra, GB, 1982) Professora Emérita (Universidade de Brasília, Professora Permanente do Programa de Pós Graduação em Ciência da Informação da Universidade de Brasília

http://dx.doi.org/10.1590/1981-5344/2460

Este artigo apresenta o crescimento da produção científica dos grupos de pesquisa das áreas de Ciência da Informação e Museologia no Brasil cadastrados no Diretório de Grupos de Pesquisa (DGP) do Conselho Nacional de Desenvolvimento Científico e Tecnológico $(C N P q)$, no que tange à evolução da produção e a variação de publicações por tipo de canal de comunicação em a) artigos científicos em periódicos, b) livros, organizados ou edições, e c) capítulos de livros, considerando o período de 1992 a 2012. Trata-se de um 
estudo quantitativo com método de análise bibliométrica, com o uso de estatística descritiva. Houve um crescimento na produção científica das áreas de Ciência da Informação e Museologia de 1992 a 2012. Embora a produção científica em livros e capítulos de livros também tenha aumentado, há predominância dos artigos científicos publicados em periódicos.

Palavras-chaves: Grupo de pesquisa; Diretório de grupos; Produção científica; Ciência da informação; Museologia.

\title{
Features and scientific production of research groups of CNPq/DGP in the areas of Information Science and Museology (1992 - 2012)
}

\begin{abstract}
This paper presents the increase of scientific production of research groups in the areas of Information Science and Museology in Brazil which are registered in the Group Directory of DGP, of National Counsel of Technological and Scientific Development (CNPq), regarding the enhancement of production and variation among types of communication channel in a) scientific papers in journals, b) books, organizations or editions; and c) book chapters, considering the period from 1992 to 2012. This is a quantitative study with bibliometric analysis method, using descriptive statistics. There was a growth in scientific production in the areas of Information Science and Museology from 1992 to 2012. Although the scientific literature in books and book chapters has also increased, there is a predominance of scientific articles published in journals.
\end{abstract}

Keywords: Research group; Group directory; Scientific production; Information Science; Museology.

Recebido em 11.06.2015 Aceito em 03.11.2015

\section{Introdução}

A tendência da ciência moderna é a colaboração e tal pressuposto reforça o valor aos estudos centrados em grupos. No Brasil, as atividades de produção de conhecimentos como resultado de pesquisa vêm sendo 
desenvolvidas por equipes de pesquisadores titulados ou em formação, organizados sob a designação de Grupos de Pesquisa (BARBOSA; SASSO; BERNS, 2009). Os grupos, quando estruturados, são caracterizados pelo compartilhamento ideológico, com condutas baseadas em normas, valores e crenças, e com relação interdependente entre os seus membros (MEADOWS, 1999, p. 80; ORRICO; OLIVEIRA, 2005).

Parte da comunidade científica brasileira que realiza suas atividades de pesquisa em grupos mantém suas composições de trabalho registradas no Diretório de Grupos de Pesquisa (DGP) do Conselho Nacional de Pesquisa (CNPq). Percebe-se um aumento do interesse em estar incluído neste repositório, talvez em decorrência da visibilidade aos pesquisadores, estudantes e técnicos, às linhas de pesquisa, e especialidades do conhecimento.

Entender um pouco mais os grupos de pesquisa de uma determinada área, sobretudo, no que se refere à sua produção científica, em termos de quantidade e canais de comunicação é importante para testemunhar como esta área tem se comportado, se tem crescido, ou se precisa ser revitalizada.

Diante deste contexto, e analisando os grupos de pesquisa das áreas de Ciência da Informação e Museologia no Brasil cadastrados no diretório de grupos do CNPq, torna-se relevante entender como se configura a produção científica dos pesquisadores e estudantes destes grupos comparando-se as publicações nos canais a) artigos científicos, b) livros e c) capítulos de livros. Temos, então, a seguinte pergunta: como evoluiu a produção das publicações científicas dos pesquisadores e estudantes dos grupos de pesquisa das áreas de Ciência da Informação e Museologia no Brasil, registrados no $\mathrm{DGP} / \mathrm{CNPq}$, nestes três canais de comunicação ao longo do tempo?

Cada canal de comunicação possui suas particularidades que influenciam os pesquisadores na escolha sobre a maneira mais conveniente para publicações de trabalhos científicos. Assim, o objetivo deste artigo é registrar o crescimento da produção científica dos grupos de pesquisa das áreas de Ciência da Informação e Museologia no Brasil cadastrados no CNPq/DGP, no que tange à evolução da produção e a variação de publicações por tipo de canal em artigos científicos, livros e capítulos de livros, considerando o período de 1992 a 2012.

As atividades de produção de indicadores quantitativos em ciência, tecnologia e inovação vêm se fortalecendo no país na última década, com o reconhecimento da necessidade, por parte dos governos Federal e Estaduais, e da comunidade científica nacional, de dispor de instrumentos para definição de diretrizes, alocação de investimentos e recursos, formulação de programas e avaliação de atividades relacionadas ao desenvolvimento científico e tecnológico no país (MUGNAINI; CARVALHO; CAMPANATTI-ORTIZ, 2006).

Vickery e Vickery (1989) já afirmavam a importância em se estudar a área de Ciência da Informação, considerando a sua contribuição para a facilitação dos processos de busca, uso, mediação e compartilhamento de 
informações científicas para a construção da cidadania, seguindo princípios multidisciplinares para a construção de conhecimentos.

Apresentar a dinâmica da produção científica dos grupos de pesquisa de uma determinada área do saber pode ajudar na compreensão de como esta área tem evoluído, ou até mesmo justificar o número de programas de pós-graduação existentes. Tem crescido o interesse de pesquisadores e especialistas por dados quantitativos que auxiliam no entendimento da dinâmica de ciência e tecnologia (SANTOS, 2003).

\section{Produção científica}

Meadows (1999) relaciona produção científica à qualidade e a quantidade do que é produzido. A produção científica das áreas de informação, no Brasil, iniciou-se na década de 70, com poucos estudos, tornando-se questão de pesquisa científica, com mais frequência, a partir de 1990.

Para Witter (2001), a história da produção científica é longa, mas esparsa e com grandes interrupções. Nos anos 60, o esforço da produção científica era esboçado mais sistematicamente como análise do conhecimento científico no exterior, enquanto no Brasil a preocupação neste sentido apenas acabara de surgir.

Observa-se que a preocupação com a investigação científica vem crescendo desde a década de 90 , fato este que pode constituir um indicador de maturidade da área (OLIVEIRA, 2004), pois representa atividade de criação, produção, busca e uso do conhecimento e é um processo coletivo dinâmico, permeado de relações e alimentado pelas publicações científicas (SILVA; PINHEIRO, 2008, p. 2). A produção científica possibilita o compartilhamento de novos conhecimentos resultantes das pesquisas realizadas, proporcionando interação, visibilidade, credibilidade, reconhecimento e prestígio nas comunidades científicas.

A produção científica divulgada em periódicos científicos é importante para a constituição da ciência no âmbito acadêmico ao tornar o artigo um poderoso veículo de disseminação da informação científica (SILVEIRA, 2012). Para Alves (1987, p. 149) a publicação, suporte básico do processo de comunicação da produção científica e cultural, transformase em forma motriz, na medida em que é recuperada e divulgada, impulsionando o desenvolvimento intelectual e realimentando o ciclo de geração de conhecimento.

Cabe ressaltar que a produção científica não se restringe aos periódicos, mas abarca todas as realizações inerentes à pesquisa, ensino e aplicação prática da ciência, de onde podem resultar serviços, métodos e tecnologias em uma atividade de extensão à sociedade (MUGNAINI; CARVALHO; CAMPANATTI-ORTIZ, 2006).

Segundo Witter (1989), a produção científica concretiza-se predominantemente sob a forma de livros, teses, dissertações, artigos publicados em revistas especializadas, podendo aparecer também como 
resumos em anais de congressos e eventos similares. A autora afirma ainda que:

a produção científica está relacionada com a atuação dos cursos de pós-graduação, quer pelo seu fazer científico, quer pelo seu papel na formação de professores e pesquisadores que irão atuar em outras entidades, universitárias ou não. Seu produto é relevante, inclusive como veículo para a mudança da dependência para a independência científica e tecnológica e, consequentemente, econômica e política (WITTER, 1989, p. 29).

A reflexão sobre a literatura de um campo do conhecimento possibilita suportes à produção científica da área, evidenciando-se a evolução de saberes e práticas relacionadas, destacando-se novos conceitos e suas respectivas definições, concretizados em domínio científico específico (BUFREM et al., 2007).

Dentre os indicadores da produção científica, Rousseau (1998) considerou o número de publicações dos grupos de pesquisa. As atividades científicas estão cada vez mais organizadas e o grupo de pesquisa representa uma forma de evolução das atividades de pesquisa.

É importante entender como se comporta a produção científica de grupos de pesquisa, pois, como sugeriu Santos (2003), trata-se de uma atividade complexa, que envolve investimento e gestão não somente para a formulação de métodos e técnicas, mas também para a compreensão de fenômenos da criação de conhecimentos e impacto científico. Por outro lado, a produção científica por vezes é resultado do esforço e da preferência individual ou de grupos, aliados a outros fatores que contribuem para a realização ou não de pesquisa, como o baixo valor dado para a atividade e as dificuldades para a divulgação dos resultados de pesquisa (BARRETO; LOPES; TOLEDO, 1997).

\section{Grupos de pesquisa e o CNPq}

Meadows (1999, p. 108) já afirmava que as atividades de Justus von Liebig na primeira metade do século XIX, da Universidade de Giessen Alemanha, caminhavam para a colaboração hierarquizada em equipes. Por outro lado, foi no final da Segunda Grande Guerra, sobretudo no que foi chamado de Big Science, e no advento das novas tecnologias, que esse método de produzir ciência de forma colaborativa tornou-se mais comum entre os pesquisadores (CRAWFORD et al., 1996).

Os dados dos grupos, abertos à ampla consulta pelo CNPq, são referentes ao censo de $2010^{1}$, pois no momento de produção deste estudo ainda estavam sendo tratados os dados do censo 2014. Tais dados apontam que o diretório de grupos possui 541 instituições registradas em

\footnotetext{
${ }^{1}$ Disponível em: <http://lattes.cnpq.br/web/dgp>. Acesso em: 29 abr. 15.
} 
sua base, e o total de grupos de pesquisa é de $27.523^{2}$. Considerando apenas as grandes áreas predominantes, as Ciências Sociais Aplicadas contêm 3.438 grupos de pesquisa (Tabela 1).

Tabela 1 - Grupos, linhas, e participantes por grande área predominante (Brasil)

\begin{tabular}{l|c|c|c|c|c|c|c|c|c}
\hline \multicolumn{1}{c|}{ Grande Área } & $\mathbf{G}$ & $\mathbf{L}$ & $\mathbf{P}$ & $\mathbf{E}$ & $\mathbf{T}$ & $\mathbf{L} / \mathbf{G}$ & $\mathbf{P} / \mathbf{G}$ & $\mathbf{E} / \mathbf{G}$ & $\mathbf{P / L}$ \\
\hline \hline Ciências Agrárias & 2.699 & 13.609 & 25.547 & 27.249 & 4.781 & 5 & 9,5 & 10,1 & 1,9 \\
\hline Ciências Biológicas & 3.108 & 14.585 & 23.390 & 32.081 & 4.638 & 4,7 & 7,5 & 10,3 & 1,6 \\
\hline Ciências Exatas e da Terra & 2.934 & 13.418 & 19.645 & 22.140 & 2.385 & 4,6 & 6,7 & 7,5 & 1,5 \\
\hline Ciências Humanas & 5.387 & 16.813 & 41.196 & 47.939 & 3.214 & 3,1 & 7,6 & 8,9 & 2,5 \\
\hline Ciências Sociais Aplicadas & 3.438 & 10.422 & 23.877 & 23.569 & 1.891 & 3 & 6,9 & 6,9 & 2,3 \\
\hline Ciências da Saúde & 4.573 & 16.728 & 34.375 & 41.446 & 6.661 & 3,7 & 7,5 & 9,1 & 2,1 \\
\hline Engenharias & 3.548 & 16.115 & 25.275 & 30.274 & 3.324 & 4,5 & 7,1 & 8,5 & 1,6 \\
\hline Linguística, Letras e Artes & 1.836 & 5.025 & 12.140 & 14.157 & 590 & 2,7 & 6,6 & 7,7 & 2,4 \\
\hline Totais & $\mathbf{2 7 . 5 2 3}$ & $\mathbf{1 0 6 . 7 1 5}$ & $\mathbf{2 0 5 . 4 4 5}$ & $\mathbf{2 3 8 . 8 5 5}$ & $\mathbf{2 7 . 4 8 4}$ & $\mathbf{3 , 9}$ & $\mathbf{7 , 5}$ & $\mathbf{8 , 7}$ & $\mathbf{1 , 9}$ \\
\hline
\end{tabular}

Fonte: CENSO CNPQ (2010). Onde: $G=$ Grupo; $L=$ Linha de Pesquisa; $P=$

Pesquisadores; $\mathrm{E}=$ Estudantes; $\mathrm{T}=$ Técnicos. $\mathrm{L} / \mathrm{G}=$ Média de Linhas de Pesquisa por Grupo; P/G = Média de Pesquisadores por Grupo; E/G = Média de Estudantes por Grupo; $P / L=$ Média de Pesquisadores por Linha.

Note-se que os grupos de pesquisa das Ciências Sociais Aplicadas apresentam, quando comparados aos grupos das demais grandes áreas, a relação mais baixa entre estudante por grupo de pesquisa $(E / G)$, e a segunda mais baixa entre linhas de pesquisa por grupo de pesquisa (L/G); apresentam ainda a terceira mais baixa entre pesquisadores por grupo de pesquisa $(P / G)$ e a terceira maior entre pesquisadores por linha de pesquisa $(P / L)$. Esses dados sugerem que os grupos são formados principalmente por pesquisadores, distribuídos em poucas linhas de pesquisa por grupo.

Especificando mais os registros do DGP para as áreas de informação, o CNPq, assim como a CAPES, trata a Museologia separadamente da Ciência da Informação. O diretório possui 174 grupos de pesquisa da Ciência da Informação (Tabela 2). A Museologia possui, de acordo com o mesmo censo, 19 grupos de pesquisa cadastrados. Juntas, estas duas áreas contêm 629 linhas de pesquisa, sendo 571 da Ciência da Informação e 58 da Museologia.

Tabela 2 - Grupos, linhas, e participantes por área do conhecimento (Brasil)

\begin{tabular}{l|c|c|c|c|c|c|c|c|c}
\hline \multicolumn{1}{c|}{ Grande Área } & G & L & $\mathbf{P}$ & $\mathbf{E}$ & $\mathbf{T}$ & $\mathbf{L} / \mathbf{G}$ & $\mathbf{P} / \mathbf{G}$ & $\mathbf{E} / \mathbf{G}$ & $\mathbf{P} / \mathbf{L}$ \\
\hline Ciência da Informação & 174 & 571 & 1325 & 1258 & 200 & 3,3 & 7,6 & 7,2 & 2,3 \\
\hline Museologia & 19 & 58 & 170 & 106 & 24 & 3,1 & 8,9 & 5,6 & 2,9 \\
\hline Totais & $\mathbf{1 9 3}$ & $\mathbf{6 2 9}$ & $\mathbf{1 4 9 5}$ & $\mathbf{1 3 6 4}$ & $\mathbf{2 2 4}$ & $\mathbf{3 , 2 5}$ & $\mathbf{7 , 7 5}$ & $\mathbf{7 , 0 6}$ & $\mathbf{2 , 3 7}$ \\
\hline
\end{tabular}

Fonte: Censo CNPq (2010). Onde: $\mathrm{G}=$ Grupo; $\mathrm{L}=$ Linha de Pesquisa; $\mathrm{P}=$

Pesquisadores; $E=$ Estudantes; $T=$ Técnicos. $L / G=$ Média de Linhas de Pesquisa por Grupo; P/G = Média de Pesquisadores por Grupo; E/G = Média de Estudantes por Grupo; $P / L=$ Média de Pesquisadores por Linha.

\footnotetext{
${ }^{2}$ Disponível em: <http://lattes.cnpq.br/web/dgp/instituicoes-participantes>. Acesso em: 29 abr. 15.
} 
Após examinados os aspectos relacionados aos grupos de pesquisa por grande área e por área específica, definem-se os seguintes procedimentos metodológicos para atingir o objetivo deste estudo.

\section{Metodologia}

Trata-se de um estudo do tipo descritivo para a explicação do objeto de estudo (GIL, 1995), neste caso, a produção bibliográfica dos grupos de pesquisa das áreas de informação do Brasil, usando métodos quantitativos, tendo como base os dados do DGP/CNPq, conforme disponíveis no período da pesquisa - janeiro de 2015.

A produção científica dos grupos de pesquisa pode ser extraída, na íntegra, dos currículos Lattes, admitindo-se dupla contagem em decorrência às coautorias entre participantes dos grupos (FERRAZ; HAYASHI; HAYASHI, 2006, p. 56). Foi possível contar com o suporte da ferramenta informatizada ScriptLattes que permite a extração e compilação automática de dados sobre: (i) produções bibliográficas, (ii) produções técnicas, (iii) produções artísticas, (iv) orientações, (v) projetos de pesquisa, por meio do código identificador único de cada usuário, de modo que podem ser feitos tratamentos de redundância, geração de grafos e classificação baseados nos índices de colaboração (MENACHALCO; CESAR JUNIOR, 2009).

Este estudo trata apenas dos dados relacionados à produção bibliográfica, conforme CNPq (artigos completos publicados em periódicos, livros publicados/organizados ou edições, e capítulos de livros publicados) compreendida no período de 1992, início do DGP do CNPq, até 2012. Para fins de análise, anos de 2013 e 2014 não foram tratados por considerar-se que ainda há obras a serem publicadas pelos periódicos e/ou atualizadas nos currículos Lattes dos pesquisadores e estudantes. Os demais dados (produções técnicas e artísticas, orientações e projetos de pesquisa) foram desconsiderados por não fazerem parte do enfoque deste estudo. Considera-se, ainda, que a integridade dos dados deste estudo está condicionada à veracidade das informações cadastradas na plataforma Lattes.

O universo deste estudo é representado pelos grupos de pesquisa das áreas de Ciência da Informação e Museologia cadastrados no DGP/CNPq e sua produção bibliográfica registrada na Plataforma Lattes, delimitando-se apenas aos pesquisadores e estudantes que os compõem, sendo que os técnicos não foram considerados no escopo do estudo. Assim, para identificar estes pesquisadores e estudantes, foi necessário recorrer ao diretório para identificar primeiro todos os grupos de pesquisa cadastrados e declarados como das áreas de Ciência da Informação e Museologia (193 grupos), dentro da grande área de Ciências Sociais Aplicadas, que por sua vez contém o total de 3438 grupos de pesquisa. Feito isso em cada grupo, que pode conter uma ou mais linhas de pesquisa, foram coletados todos os nomes completos dos pesquisadores e estudantes (1495 pesquisadores e 1364 estudantes) para, então, fazer 
buscas com cada um destes nomes na plataforma Lattes com a finalidade de copiar o código identificador único para processamento na aplicação ScriptLattes. Embora os dados tenham sido coletados em 2015, tratam-se do resultado do censo realizado ainda em 2010, que é o último resultado divulgado pelo CNPq.

A análise dos dados foi feita por meio de método bibliométrico com aplicação de estatística básica e descritiva, pertinente para construir indicadores sobre a dinâmica da informação científica (PRITCHARD, 1969), usando as variáveis "canal de comunicação" (artigos científicos, livros e capítulos de livros) e "quantidade de obras por ano de publicação".

\section{Resultados: produção científica dos grupos de pesquisa $(1992-2012)$}

A Tabela 3 concatena a quantidade anual de publicações de artigos, livros e capítulos de livros, entre o ano de 1992 e o ano de 2012, pelos grupos de pesquisa das áreas de Informação (neste caso, Ciência da Informação e Museologia), cadastrados no CNPq/DPG, e apresenta as frequências absolutas e relativas por ano.

Tabela 3: Publicações de Ciência da Informação e Museologia por canal $(1992-2012)$

\begin{tabular}{|c|c|c|c|c|c|c|c|}
\hline \multirow{2}{*}{ ANO } & \multicolumn{2}{|c|}{ ARTIGO } & \multicolumn{2}{|c|}{ LIVRO } & \multicolumn{2}{|c|}{ CAPÍTULO } & \multirow{2}{*}{ Total/Ano } \\
\hline & FA & FRc & FA & FRc & FA & FRc & \\
\hline 1992 & 110 & $59 \%$ & 49 & $26 \%$ & 29 & $15 \%$ & 188 \\
\hline 1993 & 154 & $75 \%$ & 38 & $18 \%$ & 14 & $7 \%$ & 206 \\
\hline 1994 & 163 & $65 \%$ & 51 & $20 \%$ & 36 & $14 \%$ & 250 \\
\hline 1995 & 230 & $68 \%$ & 61 & $18 \%$ & 49 & $14 \%$ & 340 \\
\hline 1996 & 262 & $75 \%$ & 46 & $13 \%$ & 43 & $12 \%$ & 351 \\
\hline 1997 & 228 & $64 \%$ & 70 & $20 \%$ & 56 & $16 \%$ & 354 \\
\hline 1998 & 285 & $64 \%$ & 85 & $19 \%$ & 74 & $17 \%$ & 444 \\
\hline 1999 & 329 & $63 \%$ & 80 & $15 \%$ & 113 & $22 \%$ & 522 \\
\hline 2000 & 407 & $64 \%$ & 87 & $14 \%$ & 141 & $22 \%$ & 635 \\
\hline 2001 & 371 & $63 \%$ & 101 & $17 \%$ & 115 & $20 \%$ & 587 \\
\hline 2002 & 429 & $63 \%$ & 93 & $14 \%$ & 158 & $23 \%$ & 680 \\
\hline 2003 & 484 & $58 \%$ & 122 & $15 \%$ & 226 & $27 \%$ & 832 \\
\hline 2004 & 587 & $64 \%$ & 102 & $11 \%$ & 224 & $25 \%$ & 913 \\
\hline 2005 & 610 & $58 \%$ & 114 & $11 \%$ & 329 & $31 \%$ & 1.053 \\
\hline 2006 & 697 & $58 \%$ & 160 & $13 \%$ & 337 & $28 \%$ & 1.194 \\
\hline 2007 & 761 & $56 \%$ & 145 & $11 \%$ & 443 & $33 \%$ & 1.349 \\
\hline 2008 & 782 & $51 \%$ & 198 & $13 \%$ & 540 & $36 \%$ & 1.520 \\
\hline 2009 & 830 & $55 \%$ & 202 & $13 \%$ & 488 & $32 \%$ & 1.520 \\
\hline 2010 & 888 & $51 \%$ & 217 & $12 \%$ & 634 & $36 \%$ & 1.739 \\
\hline 2011 & 1.090 & $54 \%$ & 250 & $12 \%$ & 665 & $33 \%$ & 2.005 \\
\hline 2012 & 1.140 & $54 \%$ & 233 & $11 \%$ & 722 & $34 \%$ & 2.095 \\
\hline Total/ Média & 10.837 & $58 \%$ & 2.504 & $13 \%$ & 5.436 & $29 \%$ & 18.777 \\
\hline
\end{tabular}

Fonte: Dados gerados pela aplicação ScriptLattes. 
Onde: ARTIGO $=$ Artigos Científicos Publicados em Periódicos; LIVRO = Livros Publicados/Organizados ou Edições; CAPÍTULO = Capítulos de Livros Publicados; $\mathrm{FA}=$ Frequência Absoluta; $\mathrm{FRC}=$ Frequência Relativa por canal.

Há uma evolução das publicações ao longo do período 1992 a 2012, com base na Tabela 3. Nesse período, o total de produção passa de 188 para 2.095, tendo como elemento impulsionador inicial a publicação dos artigos científicos, mas já a partir de 2002, livros e capítulos de livros também decolam. Com base nos dados da Tabela 3, foi construído o Gráfico 1 que mostra com mais clareza a participação anual da produção científica levantada por canal de comunicação: artigos científicos publicados em periódicos, livros e capítulos de livros, considerando o período de 20 anos (1992 - 2012).

Gráfico 1: Tendências de publicações por canal de comunicação em relação aos anos de 1992 a 2012, referentes às áreas de Ciência da Informação e Museologia

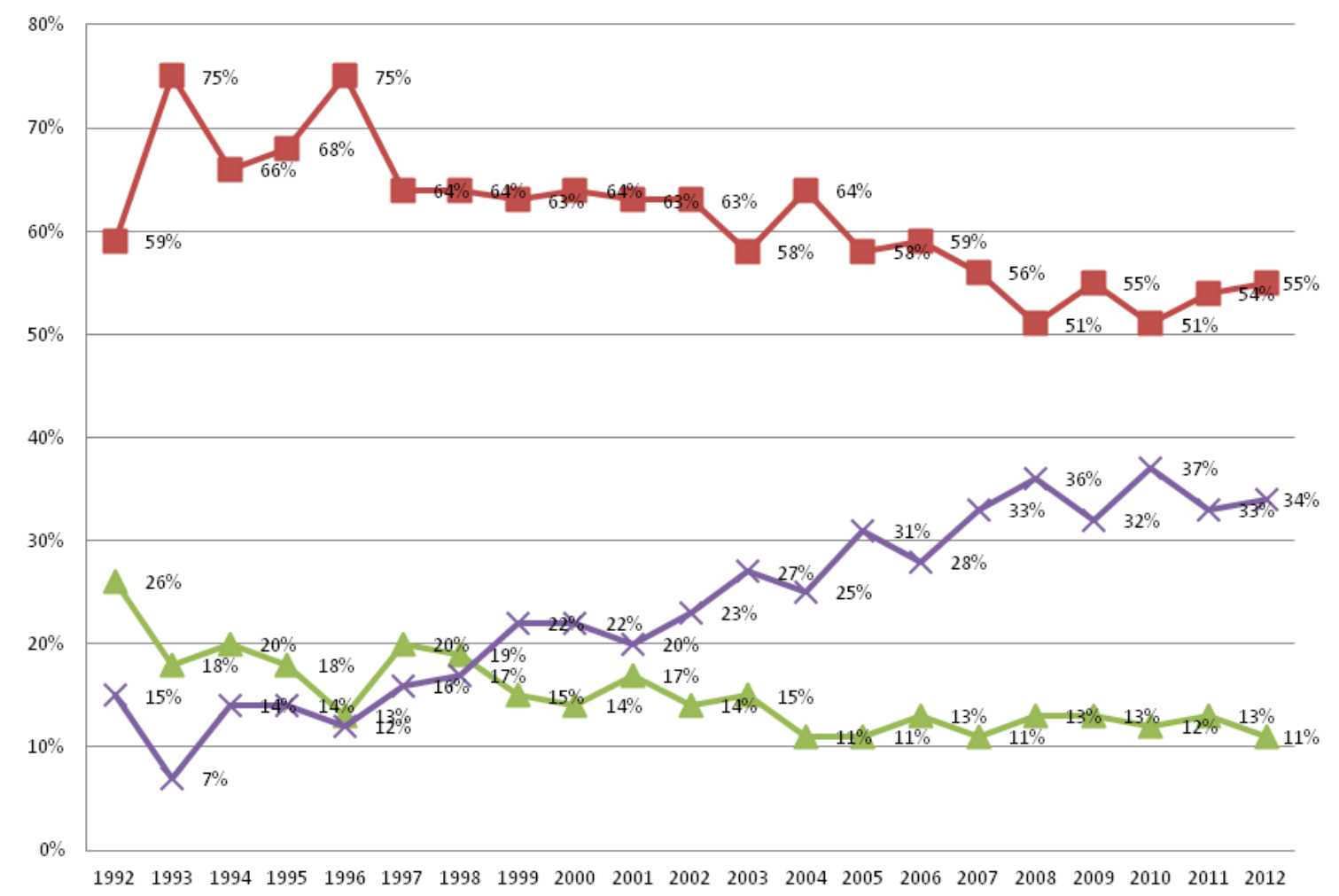

$\longrightarrow$ ARTIGO $\longrightarrow$ LIVRO $\longrightarrow$ CAPITULO

Fonte: Dados gerados pela aplicação ScriptLattes.

Em números absolutos, como apresentado na Tabela 3, houve crescimento anual quando comparado o número de publicações por canal de comunicação estudado. Já o Gráfico 1, apresenta a participação relativa anual de cada tipo de produção bibliográfica. A produção científica 
publicada em capítulos de livros evoluiu e isso se define entre 2007 e 2008 , quando alcança a frequência relativa de $37 \%$.

As estatísticas descritivas cunhadas na Tabela 4 permitem uma análise mais detalhada da produção científica total e de suas modalidades. Em 20 anos, a produção total cresceu $1.014 \%$, passando de 188 para 2.095 publicações, média anual de 894 e taxa anual de crescimento de $13 \%$. A publicação de artigos, por sua vez, cresceu $936 \%$, saltando de 110, em 1992, para 1.140, em 2012, com uma média de 516 publicações anuais e incremento exponencial de $12 \%$ ao ano. Já a publicação de livros, organizados ou edições, aumentou no período $376 \%$, passando de 49 para 233, com uma média de 119 publicações anuais e incremento exponencial de $8 \%$ ao ano.

Tabela 4 - Estatísticas descritivas sobre as publicações das áreas de Ciência da Informação e Museologia entre 1992 e 2012

\begin{tabular}{l|c|c|c|c|c|c|c}
\hline \multirow{2}{*}{$\begin{array}{l}\text { Tipos de } \\
\text { Publicações }\end{array}$} & \multicolumn{2}{|c|}{$\begin{array}{c}\text { Ano inicial e final } \\
\text { da série }\end{array}$} & \multicolumn{2}{|c|}{ Publicações anuais } & \multicolumn{2}{c}{ Taxa de variação } \\
\cline { 2 - 8 } & $\mathbf{1 9 9 2}$ & $\mathbf{2 0 1 2}$ & Média & Máxima & Mínima & $\begin{array}{c}\text { Exponencial } \\
\text { anual }\end{array}$ & $\begin{array}{c}\text { Entre } \\
\text { (1992-2012) }\end{array}$ \\
\hline \hline ARTIGO & 110 & 1.140 & 516,05 & 1140 & 110 & $12 \%$ & $936 \%$ \\
\hline LIVRO & 49 & 233 & 119,24 & 250 & 38 & $8 \%$ & $376 \%$ \\
\hline CAPITULO & 29 & 722 & 258,85 & 722 & 14 & $17 \%$ & $2390 \%$ \\
\hline TOTAL & 188 & 2.095 & 894,14 & 2095 & 188 & $13 \%$ & $1014 \%$ \\
\hline
\end{tabular}

Fonte: Dados da pesquisa.

A Tabela 4 apresenta um incremento médio anual de $13 \%$ das publicações das áreas de Ciência da Informação e Museologia entre 1992 e 2012. Embora a quantidade de artigos científicos publicados em periódicos seja bem maior que a quantidade de capítulos de livros publicados, verifica-se que este segundo canal tem o percentual de crescimento mais de duas vezes maior $(2390 \%)$ do que o percentual dos artigos científicos, passando de 29 capítulos publicados em 1992 para 2095, em 2012, com uma média de 259 publicações anuais e incremento exponencial de $17 \%$ ao ano. Ainda assim, a quantidade de artigos científicos publicados em periódicos representa sempre mais de $50 \%$ da produção científica das áreas de Ciência da Informação e Museologia. Em seguida, são introduzidos outros comentários acerca de cada canal de comunicação estudado.

\section{a) Artigos completos publicados em periódicos}

A Tabela 2 mostra que artigos científicos publicados em periódicos são mais numerosos que os demais canais durante todo o período levantado. Conforme Gráfico 1, excluindo-se os itens sem ano que são aqueles registros de artigos completos cadastrados mas que não tiveram o ano de publicação informados, percebe-se que houve uma evolução sistemática com pico evidente entre 2011 e 2013 (2011 = 1090; $2012=$ $1140 ; 2013=1075)$, com tendência de crescimento. Considerando-se o período entre 1992, que registrou 110 artigos, a 2012, com 1140 artigos, 
houve um aumento de 1030 artigos, ou 936\% (Tabela 3), com média de 51 artigos por ano.

b) Livros publicados/organizados ou edições

O número de livros publicados, organizados ou edições tem seu ápice entre 2010 e 2012. Pequenas variações são observadas entre os anos de 1992 e 2009, com alternâncias entre aumento e diminuição do número de publicações da natureza avaliada. Conforme o Gráfico 1, a partir de 1999 em diante há uma mudança significativa, pois há mudança de posicionamento entre livros e capítulos de livros. Neste ano, o percentual de publicação de livros cai para $15 \%$ e o percentual de publicação em capítulos de livros sobe para $22 \%$ e não é mais ultrapassado.

\section{c) Capítulos de livros publicados}

Com uma relação muito próxima aos artigos completos publicados em periódicos, conforme os dados da Tabela 2 e os percentuais de produção apresentados no Gráfico 1, observa-se um crescimento significativo da quantidade de publicações como capítulos de livros, enquanto a publicação em livros permanece linear. Em 1993, houve a menor quantidade de publicações (14 capítulos) e o ano de 2012 figura a maior quantidade de capítulos de livros publicados (722 capítulos), um crescimento de 708 itens, ou 5157\%. Tomando-se ainda como base o ano de 2012, e conforme a Tabela 1 , a frequência relativa da quantidade de publicações por ano em capítulos de livros é superior $(11,4 \%)$ ao ser comparada aos demais canais e no mesmo ano de referência.

\section{Considerações finais}

Este estudo teve como objetivo apresentar algumas características da produção científica dos grupos de pesquisa das áreas de Ciência da Informação e Museologia e que fazem parte do CNPq/DGP, considerando o período de 1992 a 2014, avaliando-se as variáveis quantidade de publicações em função do tempo e canais de publicação, estes limitados a periódicos científicos, livros e capítulos de livros.

A produção científica dos grupos de pesquisa das áreas de Ciência da Informação e Museologia têm crescido ao longo dos anos. Houve uma evolução cadenciada do número de publicações de artigos completos. Em 1998, a quantidade de publicações em capítulos de livros ultrapassa a quantidade de publicações em livros e este cenário se mantém constante até 2012. A partir de 2007, a quantidade de publicações por ano continua a crescer, mas não há muita variação da quantidade de publicações entre os canais estudados (artigos, livros e capítulos de livros).

Os cinco últimos anos representam mais de $50 \%$ da produção científica das áreas de Informação no Brasil, pelo menos, no que se refere às áreas de Ciência da Informação e Museologia. Há uma linha regular e ascendente sobre a quantidade de publicações em artigos científicos, 
livros e capítulos de livros em função do período de existência do diretório de grupos de pesquisa do CNPq. Os dados mostram que os trabalhos dos grupos de pesquisa das Áreas de Informação no Brasil têm configurado uma crescente produção científica, quando observadas as publicações em periódicos científicos e livros, incluindo os capítulos.

Para as áreas de Ciência da Informação e Museologia, os dados apontam que, quando comparado o ano de 1992 ao ano de 2012 há um crescimento de $936 \%$ da quantidade de artigos científicos publicados em periódicos; $376 \%$ de aumento da quantidade de livros publicados, considerando o mesmo período; e mesmo apresentando a quantidade relativa bem inferior aos artigos científicos, proporcionalmente, os capítulos de livros contam com um crescimento de $2390 \%$.

Como limitação deste estudo, não foi possível separar livros de coletâneas, para uma análise mais detalhada, e também as obras publicadas em anais não foram consideradas.

Diante dos dados deste estudo, é possível afirmar que as áreas de Ciência da Informação e Museologia têm aumentado o número de publicações ao longo do tempo, preferencialmente por artigos científicos. Em 1992, o uso destes canais, em termos de quantidade de obras publicadas, era próximo. Ao longo do tempo, as quantidades de publicações entre os canais canal se distanciam, mas todos apresentam aumento, ainda que a quantidade de artigos publicados em periódico científico seja mais numerosa. Assim, os dados apontam um crescimento significativo na produção científica das áreas de Ciência da Informação e Museologia, no que se refere às publicações de artigos científicos completos, livros e capítulos de livros, mas não indica que há mudança de preferência pelo canal, pois os dados mostram a predominância dos periódicos científicos durante o período e para o universo estudados.

\section{Referências}

ALVES, M. A. M. A Biblioteca Nacional, banco de dados da produção científica e cultural brasileira. In: Seminário Nacional de Bibliotecas Universitárias, 5., Porto Alegre, 1987. Anais... Porto Alegre: UFRGS, 1987. v.1. p.149-166.

BARBOSA, S. de F.F.; SASSO, G.T.M.D; BERNS, I. Enfermagem e tecnologia: Análise dos grupos de pesquisa cadastrados na plataforma Lattes do CNPq. Texto \& Contexto - Enfermagem, Florianópolis, v. 18, $\mathrm{n}$. 3, p.443.448, 2009.

BARRETO, A. R.; LOPES, C.; TOLEDO, N. O. Novas formas de relacionamento entre os trabalhadores do conhecimento de Universidades. In: XVIII Congresso Brasileiro de Biblioteconomia e Documentação, 18, Anais... São Luís: Collecta, 1997.

BRASIL. Conselho Nacional de Desenvolvimento Científico e Tecnológico. Censo 2010. Disponível em: <http://lattes.cnpq.br/web/dgp>. Acesso em: 29 abr. 15. 
BUFREM, L. S. et al. Produção científica em Ciência da Informação: análise temática em artigos de revistas brasileiras. Perspectivas em Ciência da Informação, v. 12, n. 1, p. 38-49, jan.-abr. 2007.

CRAWFORD, S. Y et al. Scientific Communication and the growth of Big Science. In: CRAWFORD, S. Y, HURD, J. M.; WELLER, A. C. From print to electronic. [S.I.]: ASIS, 1996. p.01-08

FERRAZ, M. C. C.; HAYASHI, M. C. P. I.; HAYASHI, C. R. M. A temática do desenvolvimento sustentável em grupos de pesquisa. Encontro de Biblioteconomia: Revista Eletrônica de Biblioteconomia, Florianópolis, v. 11 , n. 21, p. 49-68, 2006.

GIL, A. P. Como elaborar projetos de pesquisa. 3. ed. São Paulo: Atlas, 1995. 159p.

MEADOWS, A. J. A comunicação científica. Brasília: Briquet de Lemos/Livros, 1999.

MENA-CHALCO, J P.; CESAR JUNIOR, R. M. ScritptLattes: na open-source knowledge extraction system from the Lattes platform. Journal of the Brazilian Computer Society, v. 4, n.1 5, p. 31-39, 2009.

MUGNAINI, R.; CARVALHO, T.; CAMPANATTI-ORTIZ, H. Indicadores de produção científica: uma discussão conceitual. In: POBLACION, D. A. et al. (Orgs.). Comunicação e produção científica: contexto e avaliação. São Paulo: Angellara, 2006. cap.12. p. 313-340.

OLIVEIRA, H. V. de. Fatores influentes na visibilidade internacional da comunicação científica de pesquisadores de instituições da Amazônia brasileira. Rev. Cult. Pará, Belém, v. 15, n. 1, p. 61-141, 2004.

ORRICO, E. G. D.; OLIVEIRA, C. I. C. de. A representação metafórica nos caminhos do conhecimento em tempos de comunicação globalizada. DataGramaZero - Revista de Ciência da Informação, v. 6, n. 5, p. 1-12, out. 2005.

PRITCHARD, A. Statistical bibliography or bibliometricas? Journal of Documentation, v. 25, n. 4, p. 348-349, 1969.

ROUSSEAU, R. Indicadores bibliométricos e econométricos para a avaliação de instituições científicas. Ciência da Informação, Brasília, v. 27, n. 2 , p. $149-158,1998$.

SANTOS, R. N. M. dos. Produção científica: por que medir? O que medir? Revista Digital de Biblioteconomia e Ciência da Informação, Campinas, v. 1, n. 1, p. 22-38, 2003.

SILVA, E. L. da; PINHEIRO, L. V. A produção do conhecimento em ciência da informação: uma análise a partir dos artigos científicos publicados na área. Intexto, Porto Alegre, v. 2, n. 19, p. 1-24, 2008.

SILVEIRA, J. P. B. A produção científica em periódicos institucionais: um estudo da revista Biblios. Encontros Bibli: revista eletrônica de 
biblioteconomia e ciência da informação, v. 17, n. 33, p. 116-133, janabr, 2012.

VICKERY, B; VICKERY, A. Information science in theory and practice. London: Bowker-Saur, 1989.

WITTER, G. P. Pós-graduação e produção científica: a questão de autoria. Transinformação, v. 1, n. 1, p. 29-37, 1989.

WITTER, G. P. Análise de produção científica. Psicologia Escolar e Educacional, v. 5, n. 1, 2001. 